\title{
Chaotic dynamics induced by space-charge waves in cyclotron resonance accelerators
}

\author{
R. Pakter and I. L. Caldas \\ Instituto de Física, Universidade de São Paulo, Caixa Postal 66318, 05389-970 São Paulo, São Paulo, Brazil \\ F. Couto, T. Caetano, and F. B. Rizzato \\ Instituto de Física, Universidade Federal do Rio Grande do Sul, Caixa Postal 15051, 91501-970 Porto Alegre,
} Rio Grande do Sul, Brazil

(Received 18 March 1996)

\begin{abstract}
In this work, we analyze the effects produced by the inclusion of space-charge waves in a Hamiltonian model for a cyclotron resonant accelerator. We find that space charges impose limits on acceleration and that these limits appear as bounding curves on the appropriate stroboscopic phase spaces. In addition, space charges create nonlinearly locked states which undergo not only the period doubling bifurcations preceding chaos, but saddle-node inverse bifurcations as well. [S1063-651X(96)11609-3]
\end{abstract}

PACS number(s): 41.75. $-\mathrm{i}, 05.45 .+\mathrm{b}$

\section{INTRODUCTION}

A promising configuration for laser acceleration of charged particles is the so called cyclotron-resonance accelerator $[1,2]$, where a coherent electromagnetic wave may transfer a large amount of energy to a beam of electrons gyrating in a guide magnetic field. This large amount of transferred energy takes place because of the autoresonance mechanism [3,4] whereby an initial wave-particle synchronism may be self-sustained throughout the accelerating period.

Transverse space-charge effects are usually believed to be of lesser importance in cyclotron accelerators because the related fields tend to be small if the particle beam is of appropriate thickness [2]. As for longitudinal space-charge effects along the accelerating length the situation is a little different. In fact, if one works with powerful microwave sources for which the vector potential of the electromagnetic field is large, velocity gradients along the acceleration length can grow to such a magnitude that beam bunching and the corresponding space charges may become appreciable.

Our intention here is to analyze the longitudinal spacecharge effects originated from velocity gradients. We shall see that even for moderate wave amplitudes and tenuous beams, electrostatic disturbances may pose some restrictions on the acceleration efficiency. What happens is that even these relatively weak space-charge fields may induce sufficient detuning that spoils autoresonant acceleration. The effect could be seen as somewhat similar to the one created by wave dispersion, where even a small deviation from the vacuum dispersion relation for the electromagnetic wave field lowers the efficiency of the device $[3,5]$.

Furthermore, we shall see that the wave amplitude can be increased only up to a certain extent to compensate the degrading effects of the space-charge wave. Above a certain threshold the system looses its stability and undergoes a transition to chaos. The transition reveals some unusual features and bifurcations that are explored here.

The paper is organized as follows: in Sec. II we introduce the model, in Sec. III we analyze its nonlinear features, including the existence of limiting curves and types of bifurcations, and in Sec. IV we conclude the work.

\section{MODEL}

Let us assume that our system operates in a steady-state regime. Therefore, what one actually has is a beam of electrons which is continuously injected into some accelerating region lying along a magnetized, say, $z$ axis which we call the longitudinal axis. We further assume that the potential vector of the electromagnetic wave field is written as

$$
-\frac{|e|}{m c^{2}} \mathbf{A}(z)= \begin{cases}0, & \text { if } z<0 \\ \sqrt{\rho}[-\hat{\mathbf{x}} \cos (k z-\omega t)+\hat{\mathbf{y}} \sin (k z-\omega t)], & \text { if } z>0\end{cases}
$$

where $\rho$ is an adimensional constant factor determining the field amplitude, $k$ is the wave vector, $\omega$ is the wave frequency, and $\hat{\mathbf{x}}, \hat{\mathbf{y}}$ are versors along the $x$ and $y$ axis, respectively. Relation (1) means that the accelerating region lies on the half-space $z>0$. One could imagine that a wave guide is placed at $z>0$ and that a beam of particles is incident from the far left, $z<0$, with an initial velocity $v_{o} \equiv \lim _{z \rightarrow-\infty} v_{z}$ where $v_{z}$ is the $z$ component of the particle velocity. We shall see that particles cannot enter the accelerating region if $v_{o}$ is smaller than a certain threshold. However, assuming that $v_{o}$ is larger than the threshold, particles do enter the $z>0$ region and are accelerated thereafter. For those particles, if the electromagnetic wave is dispersionless and if space-charge effects are discarded, the possibility exists of 
autoresonance acceleration; it suffices to tune the wave frequency such that $\omega-k v_{o}^{+}=\Omega_{c} / \gamma$, where $v_{o}^{+}$is the longitudinal velocity at $z=0^{+} . \gamma$ is the relativistic factor and $\Omega_{c}=|e| B_{o} / m c$ is the nonrelativistic cyclotron frequency with $e$ as the electron charge, $m$ as its rest mass, $B_{o}$ as the magnitude of the guide magnetic field, and $c$ as the speed of light.

If the previous conditions are not fulfilled, acceleration is limited. However, a variety of alternatives have been presented in previous papers to compensate dispersive effects naturally found in real systems $[3,5]$. Therefore, considering that effects due to dispersion can be cured, in the present work we shall focus only on space-charge effects. In other words, we will be assuming $\omega / k=c$ throughout.

The adimensional Hamiltonian $H$ describing the temporal dynamics of an individual particle in the dispersionless case $\omega=c k$ may be written in the form (see Ref. [5] for details)

$$
H=-\omega I+\sqrt{1+2 I+\left(P_{z}+\omega I\right)^{2}+2 \sqrt{2 \rho I} \cos \theta+\rho}-\phi,
$$

where $P_{z}$ is the canonical momentum related to the kinetic momentum $p_{z}$ by $p_{z}=P_{z}+\omega I$, and where we include the adimensional electrostatic potential $\phi$, expressed in terms of the real potential as $\phi=|e| \phi_{\text {dimensional }} / m c^{2}$. The relativistic factor $\gamma$ is precisely given by

$$
\gamma=\sqrt{1+2 I+\left(P_{z}+\omega I\right)^{2}+2 \sqrt{2 \rho I} \cos \theta+\rho}=H+\omega I+\phi,
$$

the pair $(I, \theta)$ is a canonical action-angle pair describing the transverse motion across the guide field $B_{o}$, and the frequency $\omega$ is normalized with respect to $\Omega_{c}$. Since the system is time independent, $H$ is a constant. Thus it proves convenient to reconstruct the Hamiltonian formalism so as to have the coordinate $z$ playing the role of time. The receipt to carry out the transformation is known [6]; it suffices to invert relation (2) writing

$$
\begin{aligned}
P_{z}(I, \theta, \phi)= & -\omega I \\
& +\sqrt{(H+\phi+\omega I)^{2}-1-2 I-2 \sqrt{2 \rho I} \cos \theta-\rho},
\end{aligned}
$$

where the positive signal of the square root is chosen so as to represent rightward moving particles. Then, the dynamical equations can be obtained from

$$
\frac{d \theta}{d z}=\frac{\partial\left(-P_{z}\right)}{\partial I}
$$

and

$$
\frac{d I}{d z}=-\frac{\partial\left(-P_{z}\right)}{\partial \theta} .
$$

The purpose of the present investigation is to augment the previous equations with the appropriate Poisson equation for the electrostatic potential $\phi$ of the space-charge field. The Poisson equation can be cast in the appropriate adimensional form

$$
\frac{\partial^{2} \phi}{\partial z^{2}}+\nabla_{\perp}^{2} \phi=n\left(z, \mathbf{r}_{\perp}\right)
$$

where $\mathbf{r}_{\perp}$ denotes the relevant transverse coordinates of the problem and $n\left(z, \mathbf{r}_{\perp}\right)$ is the adimensional particle density given by $n=4 \pi|e| n_{\text {dimensional }} / \Omega_{c}^{2} m c$. At this point we assume that the longitudinal bunching resulting from longitudinal gradients is sufficiently intense that

$$
\kappa \equiv\left(\frac{1}{\phi} \frac{\partial^{2} \phi}{\partial z^{2}}\right)^{1 / 2}>\frac{1}{L_{t}}
$$

with $L_{t}$ as the typical transverse scale of the system. $\kappa$ is thus to be seen as some sort of longitudinal wave vector. Assumption (8) is strong. However it simplifies the analysis and its range of validity seems to be adequate enough to justify its use, as shall be discussed below. Let us write the electrostatic field as $\phi=\phi_{o}+\hat{\phi}$ where $\phi_{o}\left(\mathbf{r}_{\perp}\right)$ is the unperturbed (by the electromagnetic wave) component of the field and $\hat{\phi}\left(z, \mathbf{r}_{\perp}\right)$ is the perturbed component. Considering the fact that $\nabla_{\perp}^{2} \phi_{o}=n_{o}\left(\mathbf{r}_{\perp}\right)$, where $n_{o}$ is the unperturbed density of the beam prior its entrance into the accelerating region $z>0$, making use of approximation (8), and averaging over the cross section of the system, one is enabled to write

$$
\frac{d^{2} \phi}{d z^{2}}=n-n_{o}
$$

where the "hat" over $\phi$ has been omitted and where references to perpendicular coordinates are no longer present; from the present point of view, in particular, $n_{o}$ and $\phi_{o}$ are constant factors. We consider a beam which propagates with velocity $v_{o}>0$ for $z<0$ and assume that at $z=0$ all the particles are injected into the accelerating region, all of them with the same action $I$ and gyrophase angle $\theta$. This last assumption is consistent with the nonadiabatic phase bunching which occurs in this kind of system when particles are injected with small values of $I$ [5], a condition assumed throughout the text. With these hypotheses in mind, it becomes clear how to write $n(z)$. Indeed, one can use the continuity equation along the $z$ axis to see that

$$
n(z)=\lambda \frac{v_{o}}{v_{z}(z)},
$$

where the parameter $\lambda=4 \pi|e| n_{o} / \Omega_{c}^{2} m c$ is a measure of the unperturbed beam density; as usual, we consider beams for which $\lambda \ll 1$.

It has been commented above that Eq. (9) is justifiable if transverse derivatives are small enough that relation (8) is fulfilled. Let us examine this point in more detail. Systems without transverse bounds like particle beams in magnetospheric systems [12] can be accurately described by the previous approximations because for those beams $L_{t}$ is large enough and the system can be seen as unidimensional.

In laboratory schemes the situation is more delicate because $L_{t}$ is limited there. However one can see that even in those cases the longitudinal approximation can yield some relevant information on the dynamics. Numerical analysis reveals that $\kappa \sim 0.1$. Since we want $\kappa L_{t} \gg 1$ to discard transverse gradients, in principle on should have $L_{t} \gg 10$ for accu- 
racy. From $L_{t}=\omega\left(L_{t}\right)_{\text {dimensional }} / c$, it follows $\left(L_{t}\right)_{\text {dimensional }}$ $\gg 10 \mathrm{c} / \omega$. Considering systems with $\left(L_{t}\right)_{\text {dimensional }} \sim 10 \mathrm{~cm}$, the last result indicates that the approximation could be expected to work well if $\omega \gg 10 \mathrm{GHz}$. In this regard, we mention that the microwave regime of present accelerators uses frequencies on the order of $10 \mathrm{GHz}$ while optical and millimetric accelerators are expected to work with much higher frequencies.

Transverse forces are altogether excluded from the theory because they are expected to be small for narrow beams. In effect, Gauss law applied to a beam of cross section $L_{t}^{2}$ shows that the transverse electric field behaves as $E_{\perp} \sim \lambda L_{t}-$ hence, the conclusion. However our estimates for $\kappa$ of current laboratory schemes indicate that even in the absence of transverse forces, transverse derivatives in the Poisson equation may become comparable to longitudinal ones in determining the electrostatic potential that produces longitudinal forces. Nevertheless, longitudinal gradients are sufficiently intense that we expect our approximations to provide qualitative true results, especially on the existence, shape, and significance of limiting curves, and on the occurrence and types of bifurcations, both topics to be studied in the next section. We shall see that it is precisely the presence of the second spatial derivatives in Eqs. (7) and (9) that lends the character of dynamical variable to the space-charge perturbations. We are currently attempting to include transverse derivatives in the Poisson equation, while still trying to preserve the general structure of the formalism - a Hamiltonian structure, as shall be seen. Appropriate results will be opportunely discussed elsewhere.

In summary, we have four first-order nonlinearly coupled equations to solve. Two of them, Eqs. (5) and (6), describe action-angle dynamics and the remaining two, obtainable from Eq. (9) as

$$
\frac{d \phi}{d z}=\lambda v_{o} \mathcal{E}(z)
$$

and

$$
\frac{d \mathcal{E}}{d z}=\frac{1}{v_{z}(z)}-\frac{1}{v_{o}}
$$

describe the space charges induced by the bunched longitudinal dynamics. As seen, the action-angle equations are derived from a Hamiltonian principle. It turns out that Eqs. (11) and (12) can be also seen as a canonical pair if Hamiltonian (4) is conveniently extended. Indeed, Eqs. (11) and (12) can be made canonical if one: (i) introduces

$$
\mathcal{P}_{z}(I, \theta, \mathcal{E}, \phi) \equiv P_{z}(I, \theta, \phi)-\frac{\lambda v_{o}}{2} \mathcal{E}^{2}-\frac{1}{v_{o}} \phi
$$

(ii) looks at $\mathcal{E}$ and $\phi$ as canonically conjugate momentum and coordinate whose dynamics is derived from

$$
\frac{d \mathcal{E}}{d z}=-\frac{\partial\left(-\mathcal{P}_{z}\right)}{\partial \phi}
$$

and

$$
\frac{d \phi}{d z}=\frac{\partial\left(-\mathcal{P}_{z}\right)}{\partial \mathcal{E}}
$$

(iii) absorbs the background electrostatic field $\phi_{o}$ into $H$ as $H+\phi_{o} \rightarrow H$; and (iv) recalls that $\gamma=(H+\omega I+\phi)$ and $v_{z}=P_{z}(H, I, \theta, \phi)+\omega I / \gamma$-as mentioned before, $\omega I+P_{z}$ is the kinetic longitudinal momentum $p_{z}$ of the particle. $\mathcal{P}_{z}$ is not explicitly dependent on $z$ as long as one does not go through $z=0$ where the electromagnetic field amplitude undergoes its jump from a vanishing value for $z<0$ to $\rho$ for $z>0$. To evaluate the constant values of the Hamiltonian $\mathcal{P}_{z}$ on both sides of $z=0$, we recall that despite the discontinuity of the wave amplitude, $I, \phi$, and $\mathcal{E}$ are continuous variables whose initial values at $z=0^{+}$are imported from the unperturbed configuration at $z<0: I \sim 0, \phi=\mathcal{E}=0$. From these values and the continuity of the variables one gets

$$
\mathcal{P}_{z}=\left\{\begin{array}{cc}
\sqrt{H^{2}-1}, & \text { if } z<0 \\
\sqrt{H^{2}-1-\rho}, & \text { if } z>0,
\end{array}\right.
$$

where $H=1 / \sqrt{1-v_{o}^{2}}$ in this case. In particular, it is seen that only particles with $v_{o}^{2}>\rho /(1+\rho)$ can be injected into the region $z>0$; particles with smaller values of $v_{o}$ would be reflected backwards at $z=0$. In addition we point out that all the nonlinear interaction occurs while $\mathcal{P}_{z}$ takes the latter value of relation (16).

The fact that there exists an underlying Hamiltonian principle governing the dynamics is of foremost relevance, since it enables us to reduce the dimensionality of the problem and to construct convenient stroboscopic plots on which the dynamics can be more easily analyzed. This shall be confirmed in the next section.

\section{ANALYSIS OF THE MODEL}

\section{A. General aspects}

Before fully embarking into the complete nonlinear analysis, let us briefly see how the system behaves when electrostatic effects are turned off. For this situation where the governing Hamiltonian is simply $P_{z}$, Eq. (4), the associated phase space is shown in Fig. 1; note that since the action undergoes a wide range of variation, we use its natural logarithm, $\ln (I)$, in the plots. The figure reveals the existence of two elliptic fixed points whose respective trapping regions are isolated from each other by straight lines. One of these straight lines goes from $I=0$ to $I \rightarrow+\infty$ at $\theta=\pi / 2$, and the other goes from $I \rightarrow+\infty$ to $I=0$ at $\theta=3 \pi / 2$. In the ideal conditions considered here, particles launched at $\theta=\pi / 2$ naturally undergo unlimited acceleration as times advances. Furthermore, one sees that even a particle launched with $\theta$ $\neq \pi / 2$ can be enormously accelerated if at injection it satisfies $I \ll 1$. In this case, the particle first migrates towards $\theta=\pi / 2$ to be afterwards accelerated as previously.

As mentioned, one limiting effect on unbounded acceleration is the presence of dispersion. The other inhibiting effect, the one we are interested in, appears when the space-charge waves are turned on. In this case the complete Hamiltonian $\mathcal{P}_{z}$ is to be used. As this Hamiltonian is two-degrees of freedom, stroboscopic maps come in order. We choose to plot 


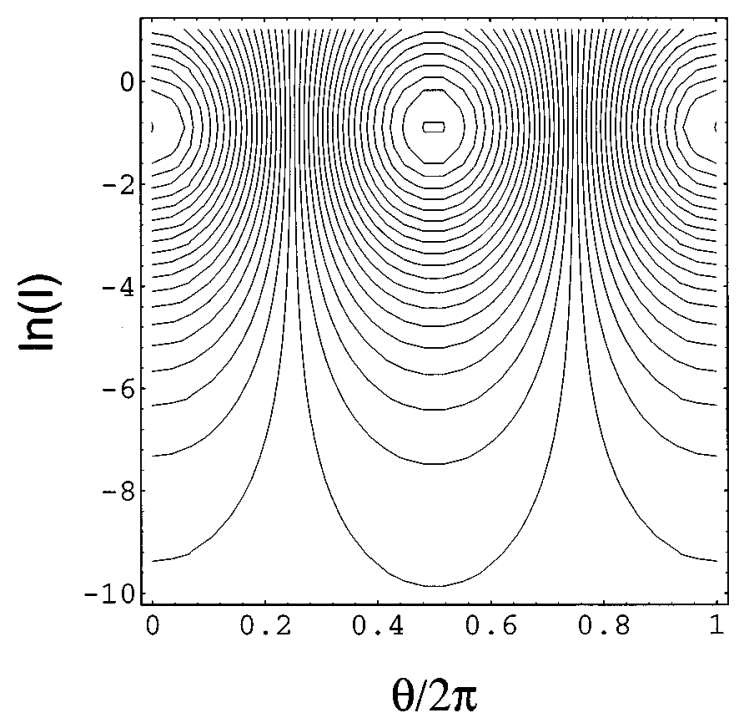

FIG. 1. Integrable phase space when space-charge fields are absent and the Hamiltonian is simply $P_{z} ; \rho=3.5 \times 10^{-6}$.

the pair $(I, \theta)$ each time $\mathcal{E}=0$ with $d \mathcal{E} / d z>0$. One relevant feature associated with our stroboscopic maps is the fact that they give rise to the appearance of some curves separating forbidden from allowed regions on the phase space. This sort of curves, called limiting curves, has been already observed in connection with relativistic systems [7]; in our present problem they can be determined as follows. One starts by considering the fact that $\mathcal{P}_{z}$ is a constant of motion. Therefore, keeping in mind that the stroboscopic plane is punctured only when $\mathcal{E}(z)=0$, one can write from Hamiltonian (13) the following relation valid on this stroboscopic plane,

$$
\phi=\phi(I, \theta, \Delta(I, \theta)),
$$

where $\Delta$ is the discriminant of the quadratic polynomial equation for $\phi$ emerging from relation (13) when $\mathcal{E}=0$. The critical condition $\Delta=0$ generates the limiting curve. In our case, its form is depicted in Fig. 2 and the possible dynamics on the stroboscopic plane develops above it. Note that for $\pi / 2<\theta<3 \pi / 2$ the corresponding $I$ vanishes. We will be mostly interested in particles injected with as low as possible initial transverse energy, which means particles whose first piercing through the stroboscopic plane is located close to the limiting curve, at $I \ll 1$ and, consequently, $\pi / 2<\theta<3 \pi / 2$. The importance of limiting curves is that they are KAM (Kolmogorov-Arnold-Moser [8]) curves to which those low energy particles stick as far as the motion is integrable there. Therefore, if a particle is launched near the limiting curve, it tends to stay near it as long as the curve is not destroyed by cascades of period doublings and chaos. Considering this feature, one can readily determine the maximum $I$ excursion merely by inspecting the maximum value of $I$ along the curve. For small values of $\rho$, a simple analysis of relation $\Delta=0$ yields $I_{\max } \sim 2 \rho^{1 / 3}$; if $\sqrt{\rho} \sim 0.001-0.01$ then $I_{\max } \sim 0.02-0.1$. Note that $I_{\max }$ is independent of $\lambda$. On the other hand we know that for small values of $\lambda$ the action grows without bounds. This apparent discrepancy is resolved

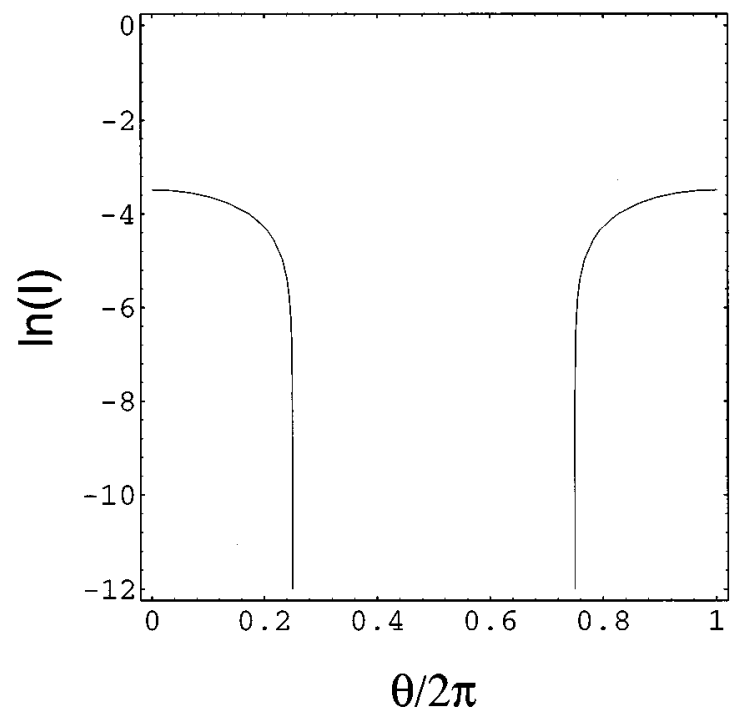

FIG. 2. Limiting curve calculated from $\Delta=0$, Eq. (17); $\rho=3.5 \times 10^{-6}$.

because as $\lambda$ is reduced, orbits no longer tend to stay close to the limiting curve which is no longer a KAM curve.

\section{B. Nonlinear analysis}

Let us now proceed to the full nonlinear analysis considering $v_{o}=0.7$ and $\lambda=0.01$ since these values for the injection velocity and average density prove to be fairly realistic both in laboratory and magnetospheric environments. For $\omega \sim 10 \mathrm{GHz}$, in particular, $\lambda=0.01$ implies in a density on the order of $10^{9} \mathrm{~cm}^{-3}$.

Focusing attention on stroboscopic plots first, note that all the initial conditions must be chosen in such way that they represent states accessible by the dynamics. In other words the initial conditions are to be chosen so as to satisfy the second relation of Eq. (16). In general we launch particles with $\theta=\pi$, with $\mathcal{E}=0$, with various values of $I$ - we denote initial values by the symbol $I_{o}$ - and with $\phi$ calculated from the conserved Hamiltonian. In Fig. 3(a) the phase space is shown for $\rho=3.5 \times 10^{-6}$. At this particular value of the wave amplitude, the phase flow is distorted in two ways. The first is connected with the appearance of the limiting curve inhibiting unbounded acceleration; if space charges are omitted the limiting curve turns into the vertical lines of Fig. 1. The other effect attached to the presence of space charge, is the occurrence of phase-locked states which appear as resonant nonlinear islands in the plots [8]. Resonant islands are the first real symptoms of nonintegrability and we should like to know how are they formed here. As it appears, they are formed as a result of the nonlinear resonance involving two typical frequencies of the system. One of the frequencies is the plasma frequency with which the density would fluctuate if the cyclotron resonance interaction were turned off-this is really what happens if particles are injected with $I$ precisely set equal to zero at $z=0$. In this case $I$ is always zero $(d I / d t=0)$ and the whole dynamics is a result of the longitudinal collective interaction of particles and space-charge waves-in other words, in this case the cyclotron resonance 
(a)
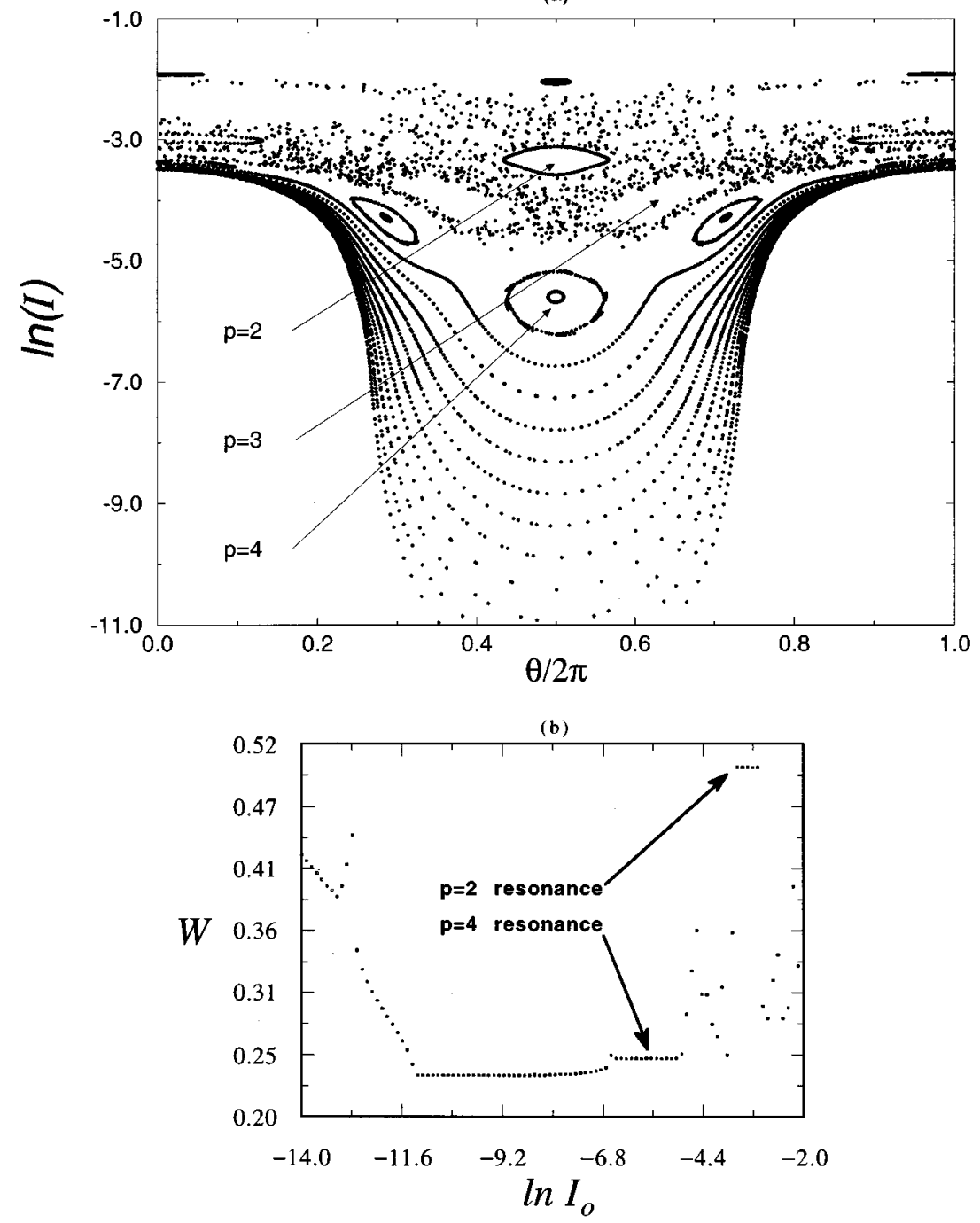

FIG. 3. (a) Stroboscopic phase space for the full system, when space-charge fields are included and the Hamiltonian is $\mathcal{P}_{z}-$ islands of the $p=4,3$, and 2 chains are indicated; (b) winding curve relative to (a); $\rho=3.5 \times 10^{-6}$. interaction is absent. The other relevant frequency is the one resulting precisely from the cyclotron resonance interaction. This frequency comes about as follows. At launching, particles are autoresonant with the wave and start by absorbing large amounts of energy. But after a while, the electrostatic potential grows driving the system out of tuning in virtue of the $\phi$-the term present in the square root of $P_{z}$ in Eq. (4). This subsequent detuning leads to periodic energy exchange and so to a finite frequency which we call the cyclotron modulation frequency. We quote that although the cyclotron modulation depends on the presence of the electrostatic potential, it is relatively independent of the value of $\lambda$, so we can really consider the system as being characterized by two independent frequencies. For the parameters used in the paper, the cyclotron modulation frequency $\omega_{\mathrm{cm}}$ is smaller than the plasma frequency, $\omega_{e}$. This suggests that the principal type of resonance could be written in the form

$$
p \omega_{\mathrm{cm}}=\omega_{e}
$$

where $p$ is an integer yielding the number of islands in the particular $p$-resonance chain. In Fig. 3(a) one can see the $p=4,3,2$ islands from bottom to top. From the figure, one is lead to think that the closer the resonant chain is to the lim- iting curve, the smaller is the frequency ratio $\omega_{\mathrm{cm}} / \omega_{e}$. This proves to be true only up to a certain extent. To examine this question in more detail, we make use of the numerically calculated winding number. The winding associated to a particular initial condition is computed as follows:

$$
W=\lim _{t \rightarrow \infty} \frac{N_{\theta}}{N_{\mathcal{E}}},
$$

where $N$ is the number of cycles of the corresponding subscripting variable counted over a very large time interval chosen such as to produce reliable statistics. From relation (19), it is readily seen that the winding number is a measure of the frequency ratio $\omega_{\mathrm{cm}} / \omega_{e}$. The complete winding curve is constructed for several initial conditions (launched similarly as in the stroboscopic analysis) and is shown in Fig. 3(b). But now the figure reveals that the winding is not a simple monotonic function of the initial action as guessed before. The winding actually is a nonmonotonic function which grows for relatively large values of $I_{o}$, but decreases if $I_{o}$ is very small. This nonmonotonicity of the winding curve has been detected in a variety of problems associated with accelerator physics [9]-[11] and implies an interesting result. The result is that at a certain point, a resonance lo- 
(a)

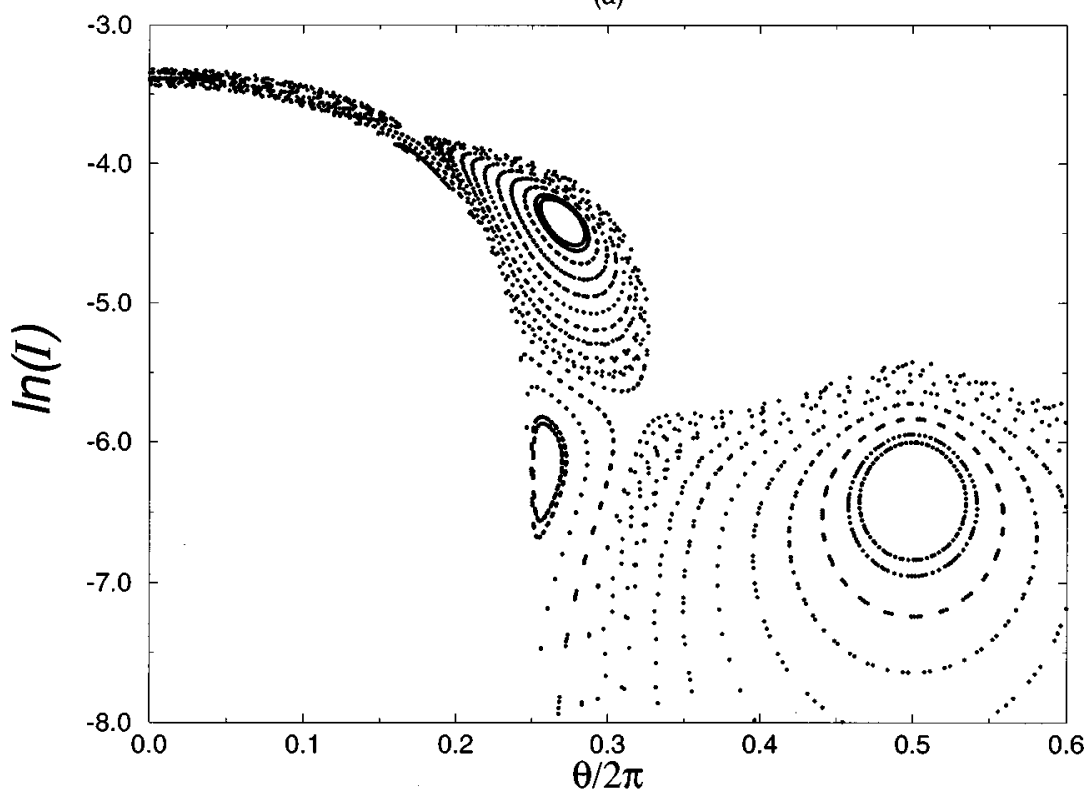

(b)

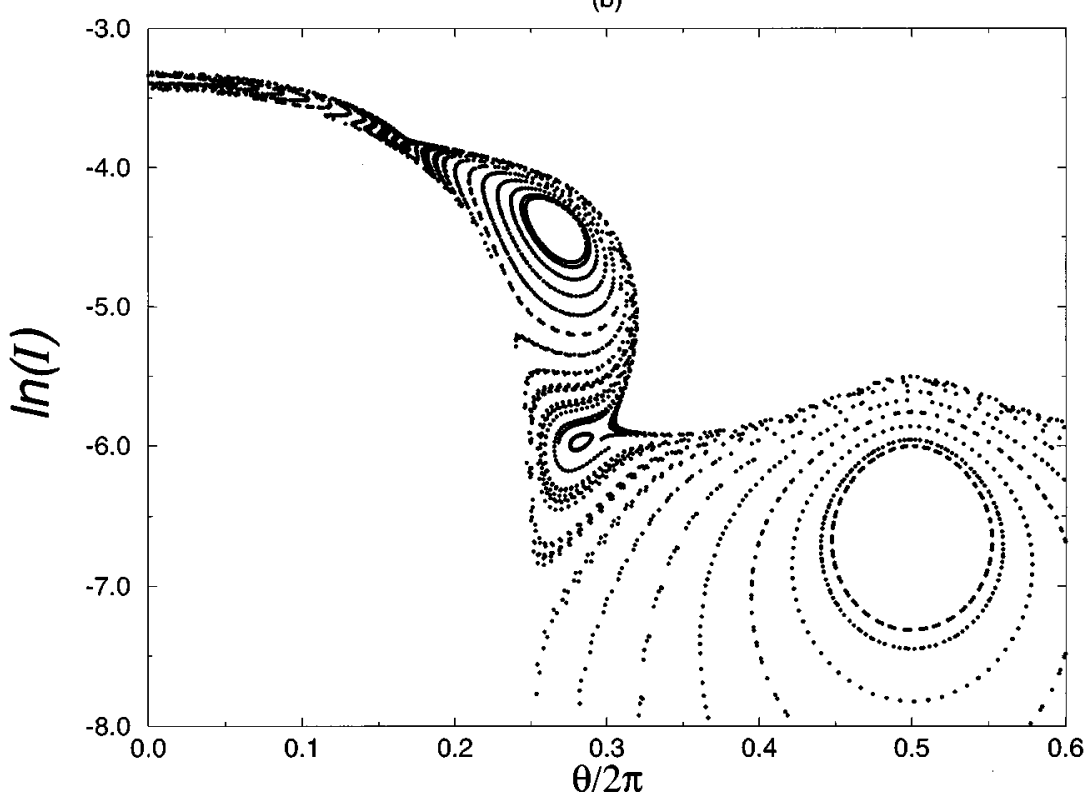

FIG. 4. The saddle-node bifurcation: (a) $\rho=4.00 \times 10^{-6} ; \quad$ (b) $\quad \rho=4.02 \times 10^{-6} ; \quad$ (c) $\rho=4.07 \times 10^{-6}$; (d) winding curve relative to the case (b). cated on the right side of the minimum of the $W$ curve can collapse against another resonance, with the same winding, but located on the left side of the minimum. This kind of bifurcation is called inverse saddle node bifurcation and we shall see how it develops in our problem. First of all we point out that resonances along a winding curve appear in the form of small plateaus. In Fig. 3(b) the resonances $p=4$ and $p=2$ can be clearly devised on the right side of the minimum. The absence of relatively large plateaus at positions where they are expected to occur, like at $W=\frac{1}{3}$, does not mean that the corresponding resonance is absent; it merely indicates that initial conditions used to construct the winding curve were launched across an hyperbolic point of the nonlinear island where the local resonance width effectively vanishes.

Returning to the analysis of bifurcations, we note that in relatively more usual cases like the nonlinear pendulum for instance, winding curves of low-dimensional Hamiltonian systems are monotonic and only period doubling bifurcations are possible. In the present situation the possibility exists of saddle nodes in virtue of the nonmonotonicity of the winding curve as mentioned before, but one should be aware that period doublings can also occur. If a resonance has not been destroyed yet and is very close to the minimum of the winding curve, a saddle-node bifurcation should be expected to occur before any period doubling. On the other hand, deep inside the monotonically increasing or decreasing sections of the winding curve the predominant type of bifurcation would be expected to be of the period doubling type.

To see how the saddle node develops here, we start by quoting that as the wave amplitude $\rho$ grows, the cyclotron modulation frequency $\omega_{\mathrm{cm}}$ grows as well and, consequently, the whole curve $W$ displaces upwards. Eventually the minimum of Fig. 3(b) ascends to the value $W=0.25$. At this precise moment the $p=4$ chain of Fig. 3(a) is expected to collide against a similar chain originally lying on the left side 
(c)
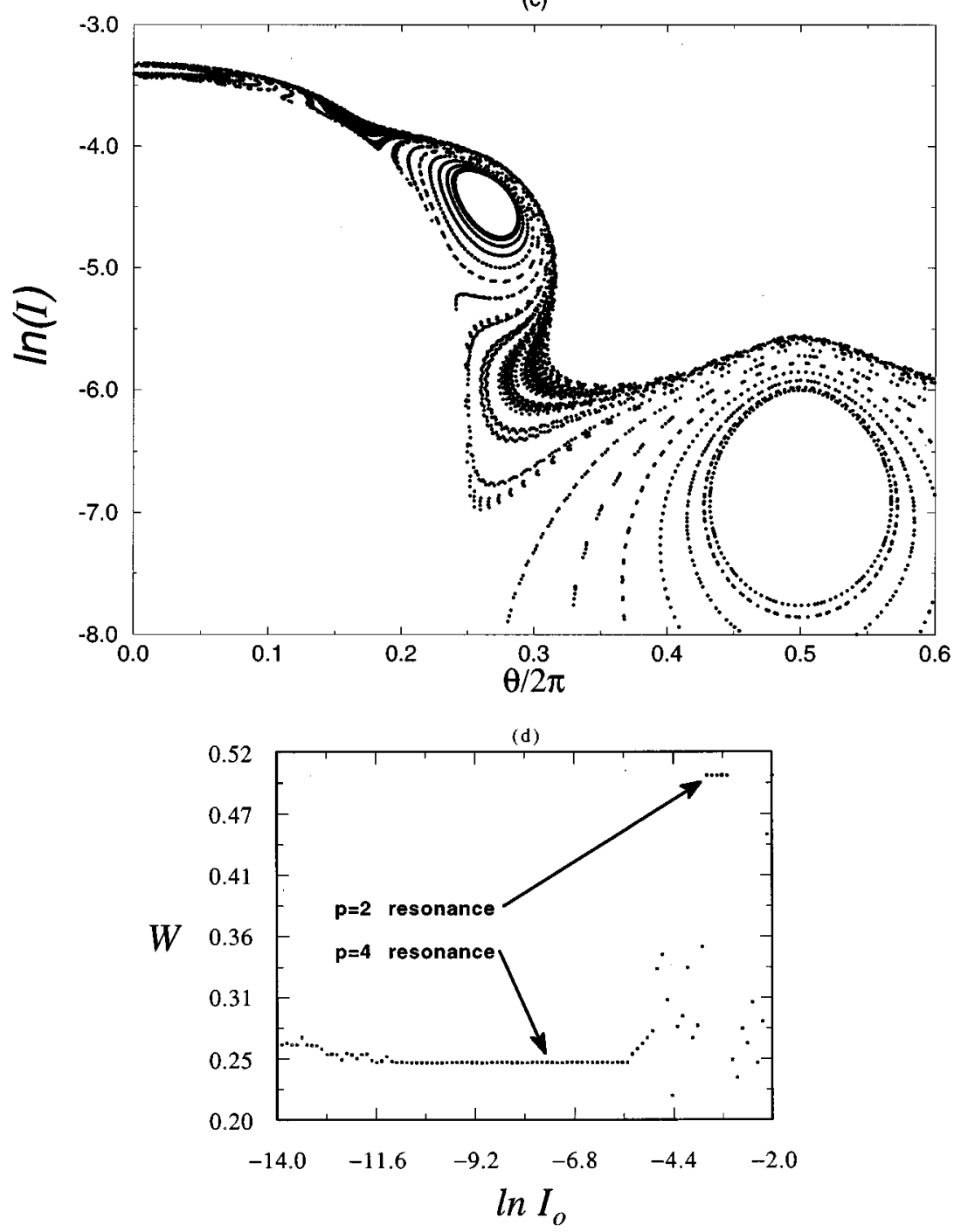

FIG. 4 (Continued).

\section{FINAL REMARKS}

In this paper we have discussed a simple model which includes electrostatic effects in a cyclotron resonance accelerating scheme. We have seen that the electrostatic field acts in two distinct ways. First of all, it sets a limiting value for the maximum accelerating efficiency. The maximum efficiency appears on a limiting curve in the appropriate stroboscopic phase space. In the second place, the presence of electrostatic fields create phase-locked states due to nonlinear resonances involving the average plasma frequency of the system and the cyclotron modulation frequency. We have also seen that if the phase-locked state is characterized by a small winding number, it lies relatively close to the limiting curve mentioned above and undergoes inverse saddle-node bifurcation. On the other extreme, when the winding number is larger, the phase-locked states are farther away from the limiting curve and undergo the more usual full period doubling cascade to chaos. While the chaotic portion of the phase space keeps away from the limiting curve, the acceleration can be expected to be regular and periodic, since particles injected close to the curve tend to stay near the curve. Only when the value of $\rho$ is large, does the chaotic portion arrive at the limiting curve, dragging the particles space. This indicates that a full cascade of period doublings has taken place. 
(a)

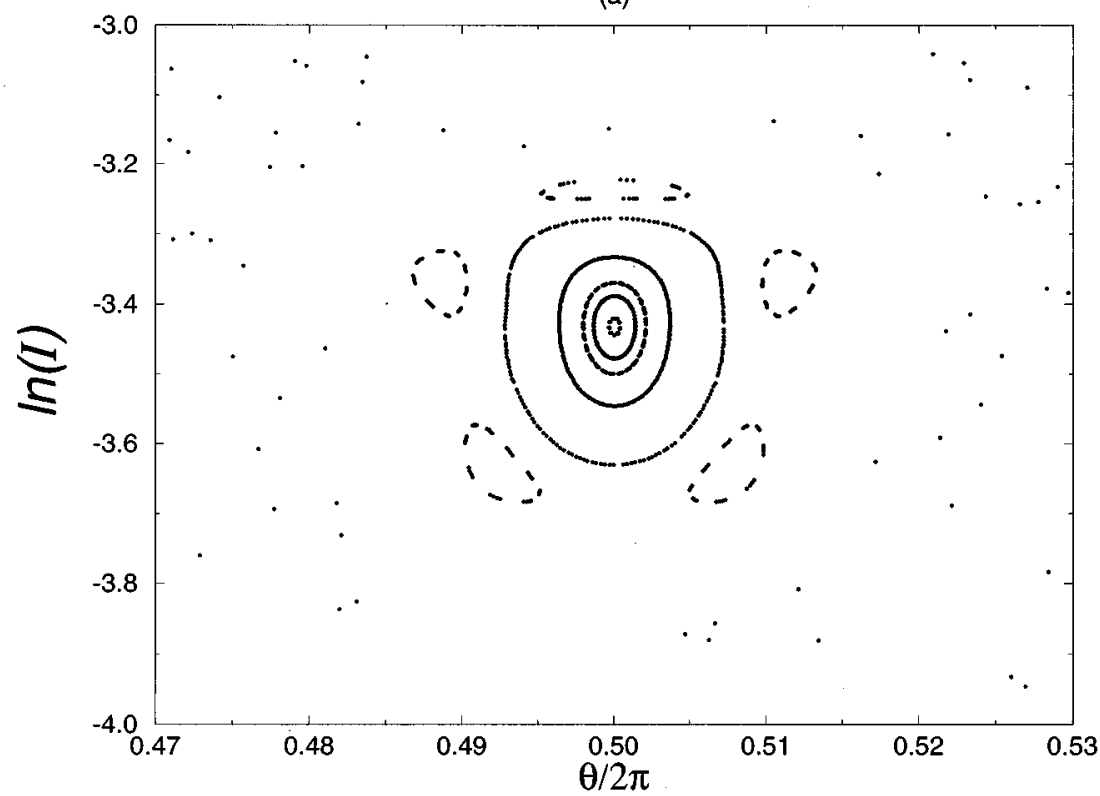

(b)

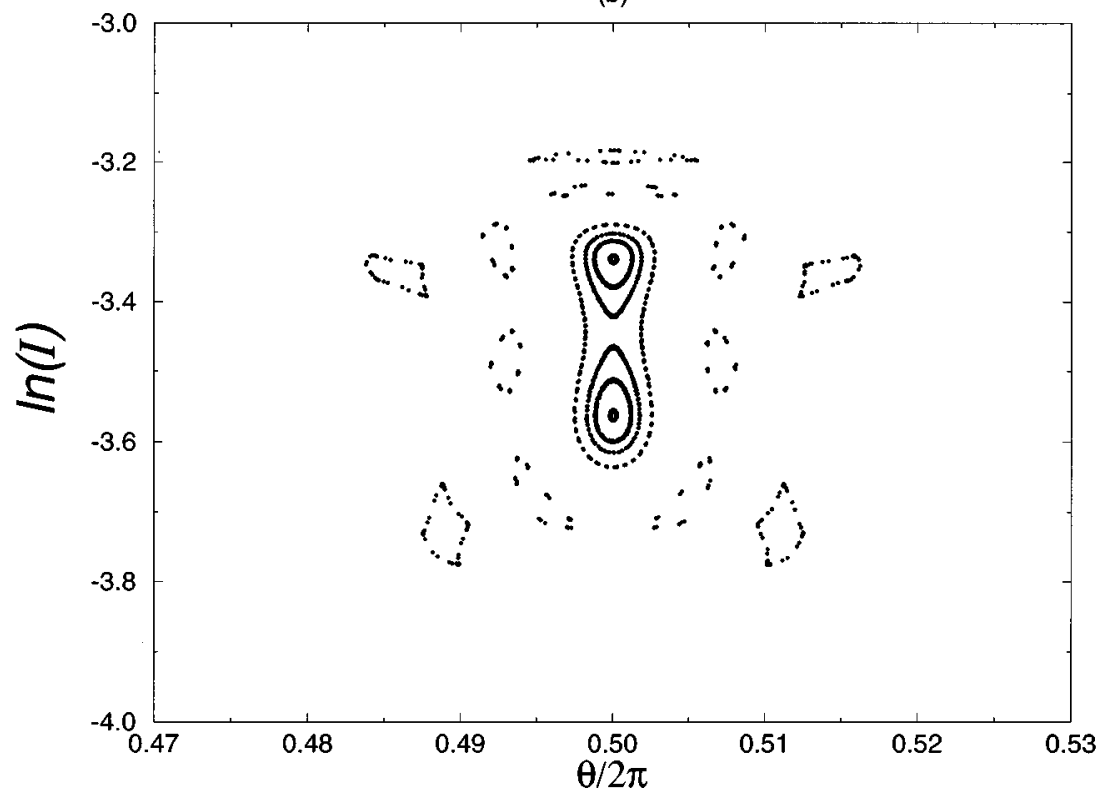

FIG. 5. The period doubling bifurcation of the $p=2$ resonance: (a) $\rho=8.0 \times 10^{-6}$; $\rho=8.5 \times 10^{-6}$. into this stochastic sea. In this case acceleration is no longer regular, although it can be very large. From these comments one sees that the wave amplitude cannot be increased forever in order to improve acceleration. For $\lambda=0.01$ and $v_{o}=0.7$, we found that chaos arrives at the limiting curve when $\sqrt{\rho} \sim 0.02$; in particular, this results in an energy gain $(\Delta \gamma)_{\max } \sim 0.5$. Chaotic activity may be difficult to detect in short laboratory devices because it takes several oscillatory periods to develop. But the bounding values arising from limiting curves are independent of the number of oscillations and can be relevant even in this case of short systems.

Our theory is accurate if $\left(L_{t}\right)_{\text {dimensional }} \gg 10 \mathrm{c} / \omega$. For thick magnetospheric beams the condition can be satisfied virtually in any range of frequencies. For narrower laboratory beams, on the other hand, the approximations are satisfied only for relatively large frequencies. Although transverse forces are still small, in the microwave range proposed for current experiments, for example, effects due to transverse derivatives in the Poisson equation can become relevant. However, even in those cases we expect the theory to provide qualitatively true results on the structure of limiting curves and on the occurrence and types of bifurcations. Transverse effects are currently under study.

\section{ACKNOWLEDGMENTS}

This work was partially supported by Financiadora de Estudos e Projetos (FINEP) and Conselho Nacional de Desenvolvimento Científico e Tecnológico (CNPq), Brazil. Numerical computing was performed on the CRAY Y-MP2E at the Universidade Federal do Rio Grande do Sul Supercomputing Center. 
[1] P. Sprangle, L. Vlahos, and C.M. Tang, IEEE Trans. Nucl. Sci. NS-30, 3177 (1983).

[2] A. Loeb and L. Friedland, Phys. Rev. A 33, 1828 (1986).

[3] C. Chen, Phys. Rev. A 46, 6654 (1992).

[4] C. Wang and J.L. Hirshfield, Phys. Rev. E 51, 2456 (1995).

[5] R. Pakter, R. Schneider, and F.B. Rizzato, Phys. Rev. E 49, 1594 (1994); R. Pakter, F. Couto, and F.B. Rizzato, ibid. 52, 4793 (1995).

[6] N. M. Kroll, P. L. Morton, and M. N. Rosenbluth, IEEE J. Quantum Electron. QE-17, 1436 (1981).
[7] G. Polymilis and K. Hyzanidis, Phys. Rev. E 47, 4381 (1993).

[8] A.J. Lichtenberg and M.A. Lieberman, Regular and Stochastic Motion (Springer, New York, 1983).

[9] G. Corso and F.B. Rizzato, Physica D 80, 296 (1995); Phys. Rev. E 52, 3591 (1995).

[10] G.A. Oda and I.L. Caldas, Chaos Solitons Fractals 5, 15 (1995).

[11] J.E. Howard and J. Humpherys, Physica D 80, 256 (1995).

[12] A.C.-L. Chian and C.F. Kennel, Astrophys. Space Sci. 97, 9 (1983). 